\title{
Sound velocities and elastic moduli of phases $I$ and $V$ of silicon at high pressures
}

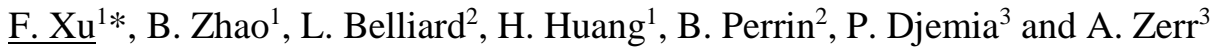 \\ ${ }^{I}$ School of Science, Wuhan University of Technology, 430070 Wuhan, China \\ ${ }^{2}$ Institut des NanoSciences de Paris (INSP), Sorbonne Université, 75005 Paris, France \\ ${ }^{3}$ Laboratoire des Sciences des Procédés et des Matériaux (LSPM)), Université Paris Nord, 93430 Villetaneuse, France
}

Keywords: silicon, high-pressure phases, sound velocities, elastic moduli

*e-mail: xufeng@whut.edu.cn

Silicon has come into wide use in semiconductor field and become the basic material due to its chemical, physical and technical properties at atmospheric pressure. Upon compression, silicon metalizes and undergoes a series of phase transitions, and thus is considered as an example elemental solid in pressure-induced phase transition studies. While most of the reported highpressure studies on silicon were focused on crystal structures of different phases [1], only few works dealt with pressure dependences of elastic moduli of Si-I, and, to our knowledge, no measurement of elastic properties of higher pressure silicon phases was reported yet.

Knowledge of sound velocities of solids at high pressures allows a better understanding of their intrinsic elastic properties, especially of $G(P)$, which is very sensitive to subtle phase transitions, defects or inhomogeneities, and permits a contrast-rich 3D tomography of non-uniform bodies. However, measurement of sound velocities at high pressures is challenging, even using standard ultrasonic techniques or more recent synchrotron-based scattering techniques. At pressures above $\sim 20 \mathrm{GPa}$, combination of picosecond laser ultrasonics (PLU) with a DAC provides access to sound velocities of opaque solids [2]. In PLU, ultrafast acoustic pulses were generated and their arrivals detected by a conventional ultrafast pump-probe setup equipped with a stabilized Michelson interferometer [3].

In present work, we investigated experimentally and theoretically pressure dependences of the longitudinal sound velocities (Figure 1) and elastic moduli (Figure 2) of Si-I and Si-V. Under compression, the starting single crystals of $\mathrm{Si}-\mathrm{I}$ underwent phase transitions and polycrystalline phases with small randomly orientated grains appeared. After the three phase transitions, the samples of $\mathrm{Si}-\mathrm{V}$ could be regarded as elastically isotropic. For the Si-I phase, the measured $V_{\mathrm{L}}(P)$ corresponds therefore to that along the $\langle 100\rangle$ direction, $V_{\mathrm{L}\langle 100\rangle}(P)$. For $\mathrm{Si}-\mathrm{V}$, the measured $V_{\mathrm{L}}(P)$ corresponds to that of an isotropic polycrystalline body $V_{\mathrm{L}(\text { avg })}(P)$ but only below 27 $\mathrm{GPa}$. The dependences $C_{11}(P)$ and $C_{12}(P)$ of $\mathrm{Si}-\mathrm{I}$, we derived from the experimental $V_{\mathrm{L}\langle 100\rangle}(P)$ using the earlier measured EOS, are in a very good agreement with previously published data and with our calculations. More important, we measured for the first time $V_{\mathrm{L}}(P)$ and $G(P)$ for polycrystalline $\mathrm{Si}-\mathrm{V}$ between $18 \mathrm{GPa}$ and $27 \mathrm{GPa}$. Above $27 \mathrm{GPa}$, we observed texturing of the polycrystalline $\mathrm{Si}-\mathrm{V}$ whose grains preferably oriented with their $c$-axes along the compressional direction in the DAC.

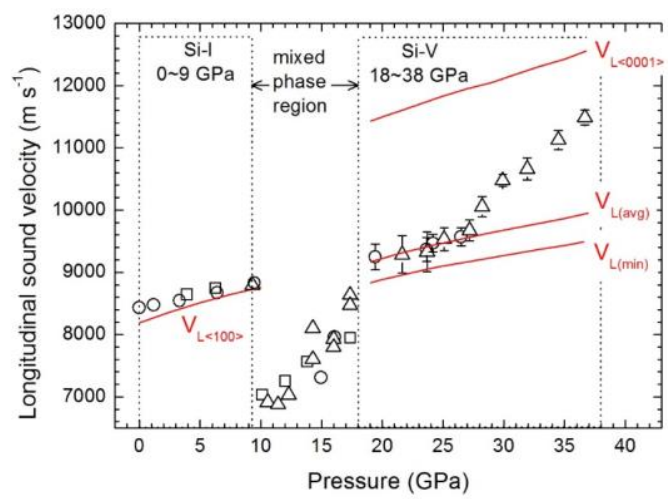

Figure 1. Longitudinal sound velocities of silicon as a function of pressure.

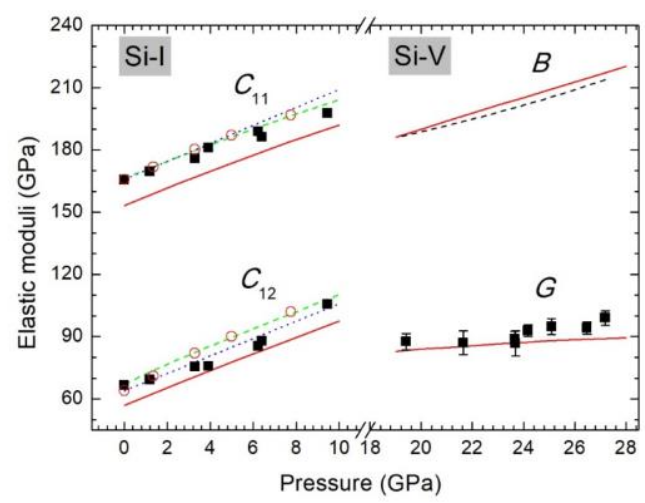

Figure 2. Pressure dependences of elastic moduli of the Si-I and $\mathrm{Si}-\mathrm{V}$ phases.

Acknowledgments: This work was supported by financial support from NSFC (projects No.41504070) and CSC (File No.201606955092). We acknowledge access to the cluster MAGI of Université Paris Nord.

[1] A. Mujica, A. Rubio, A. Munoz, R. J. Needs, Rev. Mod. Phys. 2003, 75, 863.

[2] F. Decremps, L. Belliard, B. Perrin, M. Gauthier, Phys. Rev. Lett. 2008, 100, 035502.

[3] F. Xu, L. Belliard, D. Fournier, E. Charron, J.-Y. Duquesne, S. Martin, C. Secouard, B. Perrin, Thin Solid Films 2013, $\mathbf{5 4 8 ,} 366$. 